\title{
Terahertz pump-probe experiment at the synchrotron light source MLS
}

\author{
A. Pohl ${ }^{1,2}$, A. Hoehl ${ }^{3}$, R. Müller ${ }^{3}$, G. Ulm ${ }^{3}$, J. Feikes ${ }^{4}$, M. Ries ${ }^{4}$, G. Wüstefeld ${ }^{4}$, S. Pavlov ${ }^{2}$, \\ and H.-W. Hübers ${ }^{1,2}$ \\ ${ }^{1}$ Institut für Optik und Atomare Physik, Technische Universität Berlin, 10623 Berlin, Germany \\ ${ }^{2}$ Institute of Planetary Research, DLR e.V., Rutherfordstrasse 2, 12489 Berlin, Germany \\ ${ }^{3}$ Physikalisch-Technische Bundesanstalt (PTB), Abbestrasse 2-12, 10587 Berlin, Germany \\ ${ }^{4}$ HZB für Materialien und Energie, Albert-Einstein-Str. 15, 12489 Berlin, Germany
}

\begin{abstract}
We have developed a pump-probe experiment utilizing broad-band coherent terahertz synchrotron radiation provided by the Metrology Light Source (MLS). The design, performance and first results obtained with the setup are presented.
\end{abstract}

\section{INTRODUCTION AND BACKGROUND}

$\mathrm{T}$ IME-resolved studies of energy relaxation processes in photoexcited materials by the so-called pump-probe technique is an important tool for example in semiconductor research. Pump-probe experiments are implemented at many free electron laser (FEL) facilities. An FEL provides narrow band, high power radiation. However, it needs tuning if frequency dependent processes are measured. Coherent $\mathrm{THz}$ synchrotron radiation (CSR) provides stable, broadband, short $(<10 \mathrm{ps})$ and powerful pulses (up to $60 \mathrm{~mW}$ average power) with high repetition rate (up to $500 \mathrm{MHz}$ ) [1]. The CSR spectrum at the Metrology Light Source (MLS) extends up to about $1.5 \mathrm{THz}$. Above that the incoherent radiation is more powerful. The MLS is the first electron storage ring with a dedicated electron optics for low-alpha operation and an optimized $\mathrm{THz}$ beamline. Both are necessary for the generation of stable high power THz CSR [2]. The emitted CSR power depends on the length and the shape of the electron bunch [3], [4]. However, pump-probe experiments utilizing the FELs tunable single color radiation suffer from relatively long acquisition times. The broadband CSR can be used to measure multiple frequency dependent processes simultaneously. Therefor the properties of CSR make it suitable for $\mathrm{THz}$ pump-probe experiments. We will report on the development of a pump-probe experiment at the MLS, the electron storage ring of the PTB.

\section{SETUP AND RESUltS}

The MLS provides Gaussian-shaped $\mathrm{THz}$ pulses with a repetition rate as low as $2 \mathrm{~ns}$. The pulse length is 1 to $15 \mathrm{ps}$ depending on the settings of the synchrotron and the average power is up to $60 \mathrm{~mW}$.

In order to generate pump-probe signals from the $\mathrm{THz}$ synchrotron pulses we utilize a Martin-Puplett interferometer type approach. As shown in Fig. 1 the divergent THz beam is collimated by means of an off axis parabolic mirror with a focal length of $450 \mathrm{~mm}$. A $45^{\circ}$-wire grid beam splitter divides the incoming horizontally polarized $\mathrm{THz}$ beam in two beams: one transmitted (pump) beam and another reflected (probe) beam. Both are reflected at the rooftop mirrors which rotate the polarization by $90^{\circ}$. When the reflected beams hit the beam splitter again on their way back, the probe beam is transmitted while the pump beam is reflected. The probe pulse follows the pump pulse with some tunable delay which is set by the delay line. The maximum delay is $2.7 \mathrm{~ns}$ and the minimum step size of the delay line corresponds to a delay well below $0.1 \mathrm{ps}$. Both pulses are focused onto the sample. After excitation of the sample by the pump pulse the time dependent pump-induced change in transmission $\Delta T(t)=T(t)-T_{0}$ ( $T$ and $T_{0}$ are the transmission with and without excitation) is measured with the probe pulse. The probe pulse is spectrally analyzed with an evacuated Fourier transform spectrometer while the pump pulse is deflected by another wire grid and dumped in a dedicated absorber.

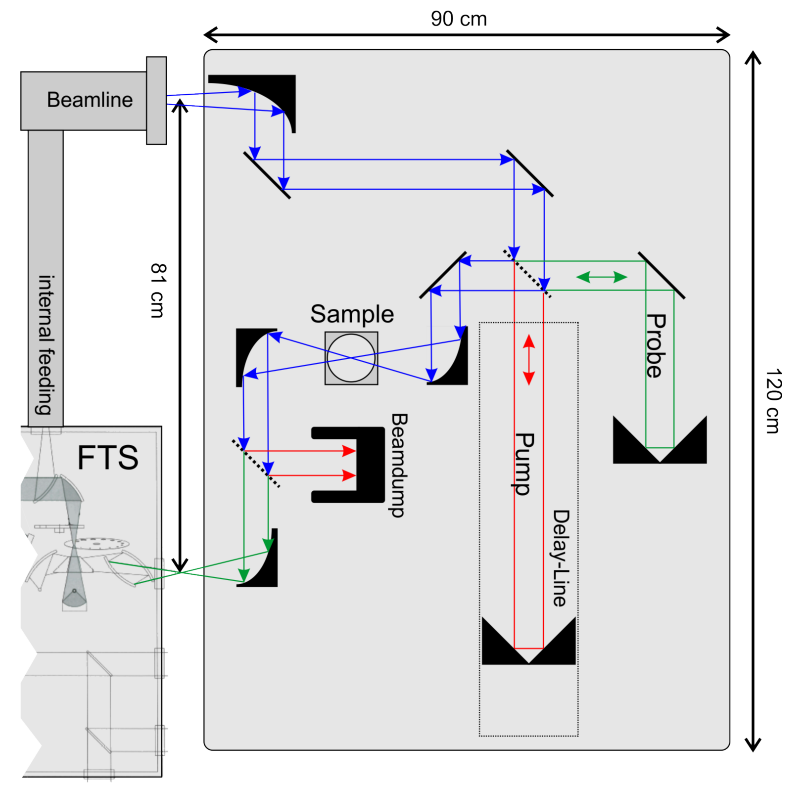

Fig. 1. Sketch of the pump-probe setup at the MLS THz beamline. 
A sample spectrum is shown in Fig. 2. The spectrum measured through the pump-probe setup is about a factor of three less powerful and hampered by water absorption caused by the $2 \mathrm{~m}$ optical path in atmosphere. This will be reduced by purging the experiment setup with dry nitrogen which will be done in an improvement step.

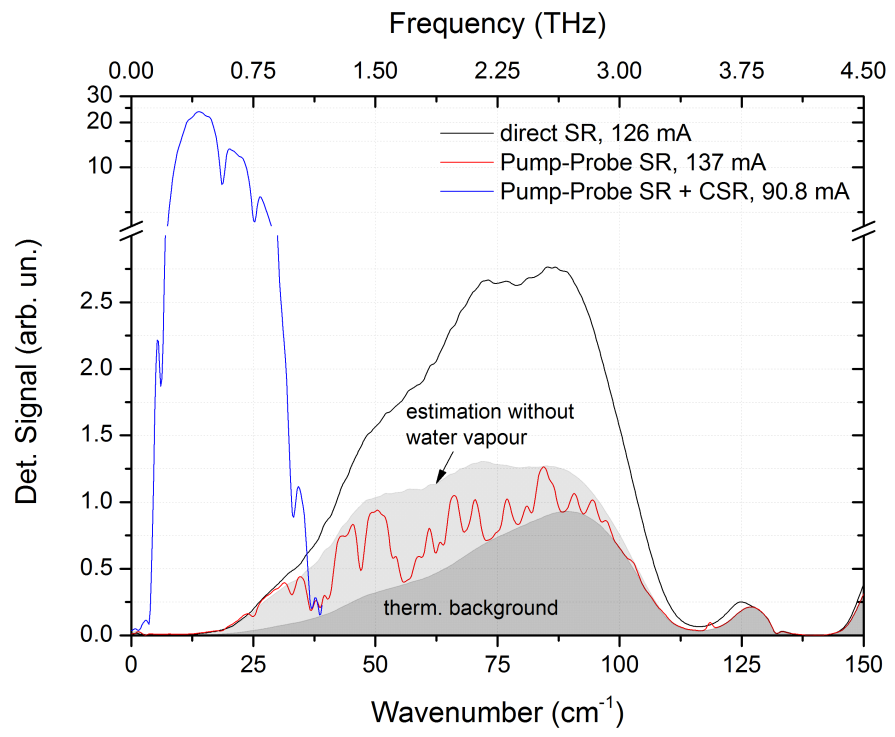

Fig. 2. Spectra of the THz signal (pump and probe) transmitted through the pump-probe setup (red and blue line) and directly (black line).

For characterization the spatial and temporal overlap of the pump and probe beams a zero-bias Schottky diode from Virginia Diodes was used. The diode was mounted at the focus position of pump and probe beam. The signal output of the Schottky diode was preamplified and then fed into a lock-in amplifier. For triggering the lock-in amplifier the MLS $500 \mathrm{MHz}$ reference clock was used. With a lock-in time constant $\mathrm{t}_{\mathrm{c}}=100 \mathrm{~ms}$ and a continuous motion of the delay line at $0.1 \mathrm{~mm} / \mathrm{s}$ the overlap area was recorded. Figure 3 shows the recorded autocorrelation signal at a beam current of $28 \mathrm{~mA}$ with the low-alpha optic at $5 \mathrm{kHz}$ tune and a fill pattern of 80 electron bunches in the storage ring. The autocorrelation is shown in Fig. 3 indicating a good overlap between pump and probe pulse.

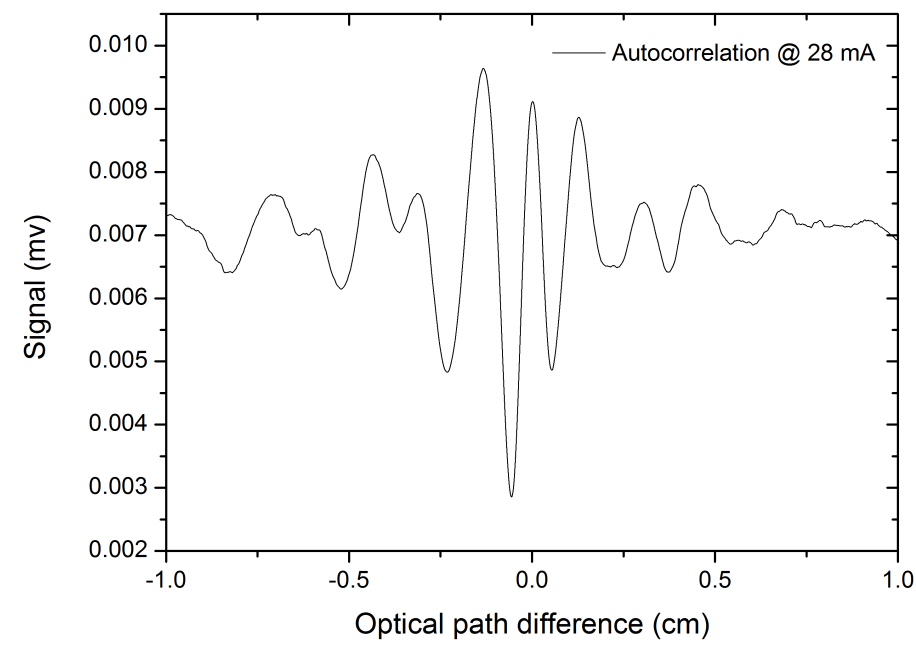

Fig. 3. Autocorrelation signal of the CSR pulse at sample position taken with a Schottky diode.

\section{CONCLUSION}

We have developed and evaluated a dedicated pump-probe experiment at the MLS of the PTB in Berlin. Key features are a broadband CSR source (up to $1.5 \mathrm{THz}$ ) with short $(<10 \mathrm{ps}$ ) and powerful pulses (up to $60 \mathrm{~mW}$ average power). The pump probe setup utilizes a Martin-Puplett interferometer type approach with a maximum delay of $2.7 \mathrm{~ns}$ at a minimum step size corresponding to $0.1 \mathrm{ps}$. The power loss in the $2 \mathrm{~m}$ optical path is at a factor of three. Improvements such as purging the experiment with dry nitrogen will reduce the strong water absorption, thus making the setup more stable with regard to temperature and water vapor fluctuations.

\section{ACKNOWLEDGEMENT}

This work has founded by the German federal Ministry of Education and Research (Grant No. 05K10KTC). The authors would like to thank J. Hessler from Virginia Diodes Inc. for providing the Schottky diode. A. Pohl acknowledges support by the Helmholtz Research School on Security Technologies.

\section{REFERENCES}

[1] J. Feikes et al., "Metrology Light Source: The first electrom storage ring optimized for generating coherent THz radiation", Phys. Rev. ST Accel. Beams. 14, 2011, 030705.

[2] R. Klein et. al., "Operation of the Metrology Light Source as a primary radiation source standard", Phys. Rev. ST Accel. Beams 11, 110701, 2008.

[3] H.-W. Hübers et. al., "Time domain analysis of coherent terahertz syncrotron radiation", Appl. Phys. Lett. 87, 184103, 2005.

[4] P. Probst et. al., " $\mathrm{YBa}_{2} \mathrm{Cu}_{3} \mathrm{O}_{7-\delta}$ quasioptical detectors for fast timedomain analysis of terahertz synchrotron radiation", Appl. Phys. Lett. 98, 2011. 